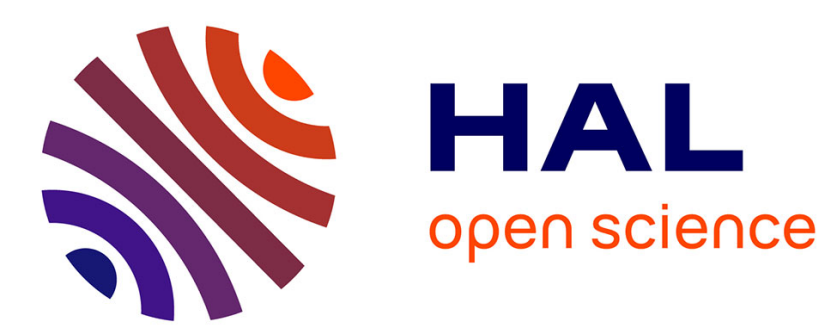

\title{
Fine-grained parallelization of a Vlasov-Poisson application on GPU \\ Guillaume Latu
}

\section{To cite this version:}

Guillaume Latu. Fine-grained parallelization of a Vlasov-Poisson application on GPU. Europar'10, HPPC Workshop, Sep 2010, Ischia, Italy. inria-00591061

\section{HAL Id: inria-00591061 \\ https://inria.hal.science/inria-00591061}

Submitted on 6 May 2011

HAL is a multi-disciplinary open access archive for the deposit and dissemination of scientific research documents, whether they are published or not. The documents may come from teaching and research institutions in France or abroad, or from public or private research centers.
L'archive ouverte pluridisciplinaire HAL, est destinée au dépôt et à la diffusion de documents scientifiques de niveau recherche, publiés ou non, émanant des établissements d'enseignement et de recherche français ou étrangers, des laboratoires publics ou privés. 


\title{
Fine-grained parallelization of a Vlasov-Poisson application on GPU
}

\author{
Guillaume Latu ${ }^{1,2}$ \\ 1 CEA, IRFM, F-13108 Saint-Paul-lez-Durance, France. \\ 2 Strasbourg 1 University \& INRIA/Calvi project \\ guillaume.latu@cea.fr
}

\begin{abstract}
Understanding turbulent transport in magnetised plasmas is a subject of major importance to optimise experiments in tokamak fusion reactors. Also, simulations of fusion plasma consume a great amount of CPU time on today's supercomputers. The Vlasov equation provides a useful framework to model such plasma. In this paper, we focus on the parallelization of a 2D semi-Lagrangian Vlasov solver on GPGPU. The originality of the approach lies in the needed overhaul of both numerical scheme and algorithms, in order to compute accurately and efficiently in the CUDA framework. First, we show how to deal with 32-bit floating point precision, and we look at accuracy issues. Second, we exhibit a very fine grain parallelization that fits well on a many-core architecture. A speed-up of almost 80 has been obtained by using a GPU instead of one CPU core. As far as we know, this work presents the first semiLagrangian Vlasov solver ported onto GPU.
\end{abstract}

\section{INTRODUCTION}

The present paper highlights the porting of a semi-Lagrangian Vlasov-Poisson code on a GPU device. The work, described herein, follows a previous study made on the LOSS code described in other papers [CLS06,CLS09,LCGS07]. A classical approach in the Semi-Lagrangian community involves the use of cubic splines to achieve the many interpolations needed by this scheme. The application we describe here, uses a local spline method designed specifically to perform decoupled numerical interpolations, while preserving classical cubic spline accuracy. In previous papers, this scalable method was described, and was benchmarked in academic and industrial simulators. Only relatively small MPI inter-processor communication costs were induced and these codes scaled well over hundreds of cores (1D and 2D domain decompositions were investigated).

Particle-in-Cell (PIC) codes are often used in plasma physics studies and they use substantial computer time at some of the largest supercomputer centers in the world. Particle-in-Cell, yet less accurate, is a most commonly used numerical method than the semi-Lagrangian one. Several papers has been published on PIC codes that harness the computational power of BlueGene and GPGPU hardwares [SDG08, $\left.\mathrm{BAB}^{+} 08\right]$ and provide good scalability. Looking for new algorithms that are highly scalable in the field of Tokamak simulations is important to mimic plasma devices with more realism. 
We will describe how to enrich the Semi-Lagrangian scheme in order to obtain scalable algorithms that fits well in the CUDA framework. In the sequel, the numerical scheme and the accuracy issues are briefly introduced and the parallelization of the main algorithm with CUDA is described. The speedup and accuracy of the simulations are reported and discussed.

\section{MATHEMATICAL MODEL}

In the present work, we consider a reduced model for two physical dimensions (instead of six in the general case), corresponding to $x$ and $v_{x}$ such as $\left(x, v_{x}\right) \in \mathbb{R}^{2}$. The $1 \mathrm{D}$ variable $x$ represents the configuration space and the $1 \mathrm{D}$ variable $v_{x}$ stands for the velocity along $x$ direction. Moreover, the self consistent magnetic field is neglected because $v_{x}$ is considered to be small in the physical configurations we are looking at. The Vlasov-Poisson system then reads:

$$
\begin{gathered}
\frac{\partial f}{\partial t}+v_{x} \cdot \nabla_{x} f+\left(E+v_{x} \times B\right) \cdot \nabla_{v_{x}} f=0, \\
-\varepsilon_{0} \nabla^{2} \phi=\rho(x, t)=q \int f\left(x, v_{x}, t\right) d v_{x}, \quad E(x, t)=-\nabla \phi .
\end{gathered}
$$

where $f\left(x, v_{x}, t\right)$ is the particle density function, $\rho$ is the charge density, $q$ is the charge of a particle (only one species is considered) and $\varepsilon_{0}$ is the vacuum permittivity, $B$ is the applied magnetic field.

Eq. (1) and (2) are solved successively at each time step. The density $\rho$ is evaluated in integrating $f$ over $v_{x}$ and Eq. (2) gives the self-consistent electrostatic field $E(x, t)$ generated by particles. Our work focuses on the resolution of Eq. (1) using a backward semi-Lagrangian method [SRBG99]. The physical domain is defined as $\mathcal{D}_{p}^{2}=\left\{\left(x, v_{x}\right) \in\left[x_{\min }, x_{\operatorname{Max}}\right] \times\left[v_{x_{\min }}, v_{x_{\operatorname{Max}}}\right]\right\}$. For the sake of simplicity, we will consider that the size of the grid mapped on this physical domain is a square indexed on $\mathcal{D}_{i}^{2}=\left[0,2^{j}-1\right]^{2}$ (it is easy to break this assumption to get a rectangle). Concerning the type of boundary conditions, a choice should be made depending on the test cases under investigation. At the time being, only periodic extension is implemented.

\section{ALGORITHMIC ANALYSIS}

\subsection{Global numerical scheme}

The Vlasov Equation (1) can be decomposed by splitting. It is possible to solve it, through the following elementary advection equations:

$$
\partial_{t} f+v_{x} \partial_{x} f=0, \quad\left(\begin{array}{ll}
\hat{x} & \text { operator })
\end{array} \partial_{t} f+\dot{v}_{x} \partial_{v_{x}} f=0 . \quad\left(\hat{v}_{x} \text { operator }\right)\right.
$$

Each advection consists in applying a shift operator. A splitting of Strang [CK76] is employed to keep a scheme of second order accuracy in time. We took the sequence $\left(\hat{x} / 2, \hat{v}_{x}, \hat{x} / 2\right)$, where the factor $1 / 2$ means a shift over a reduced time step $\Delta t / 2$. Algorithm 2 shows how the Vlasov solver of Eq. (1) is interleaved with the field solver of Eq. (2). 


\subsection{Local spline method}

Each 1D advection (along $x$ or $v_{x}$ ) consists in two substeps (Algorithm 1). First, the density function $f$ is processed in order to derive the cubic spline coefficients. The specificity of the local spline method is that a set of spline coefficients covering one subdomain can be computed concurrently with other ones. Thus, it improves the standard approach that unfortunately needs a coupling between all coefficients along one direction. Second, spline coefficients are used to interpolate the function $f$ at specific points. This substep is intrinsically parallel wether with the standard spline method or with the local spline method: one interpolation involves only a linear combination of four neighbouring spline coefficients.

In Algorithm 1, $x^{o}$ is called the origin of the characteristic. With the local spline method, we gain concurrent computations during the spline coefficient derivation (line 2 of the algorithm). Our goal is to port Algorithm 1 onto GPU.

\begin{tabular}{|c|c|}
\hline & $\begin{array}{l}\text { Algorithm 2: One time step } \\
\text { Input : } f_{t} \\
\text { Output: } f_{t+\Delta t}\end{array}$ \\
\hline Algorithm 1: Advection in $x$ dir., $d t$ time step & // Vlasov solver, part 1 \\
\hline Input $: f$ & 1 1D Advection, operator $\frac{\hat{x}}{2}$ on $f(., ., t)$ \\
\hline $\begin{array}{c}\text { Output: } f \\
1 \text { forall } v_{x} \text { do }\end{array}$ & // Field solver \\
\hline $2 \quad a(.) \leftarrow$ spline coeff. of sampled function $f\left(., v_{x}\right)$ & 2 Integrate $f(., ., t+\Delta t / 2)$ over $v_{x}$ \\
\hline $3 \quad$ forall $x$ do & $\begin{array}{l}3 \\
4\end{array}$ Compute $\Phi_{t+\Delta t / 2}$ with Poisson solver \\
\hline $\begin{array}{ll}4 & x^{0} \leftarrow x-v_{x} . d t \\
5 & f\left(x, v_{x}\right) \leftarrow \text { interpolate } f\left(x^{0}, v_{x}\right) \text { with } a(.)\end{array}$ & $5 \quad$ using $\rho(., t+\Delta t / 2)$ \\
\hline & $\begin{array}{l}\left.6 \text { 1D Advection, operator } \hat{v}_{x} \text { (use } \Phi_{t+\Delta t / 2}\right) \\
7 \text { 1D Advection, operator } \frac{\hat{x}}{2}\end{array}$ \\
\hline
\end{tabular}

\subsection{Floating point precision}

Usually, semi-Lagrangian codes make extensive use of double precision floating point operations. The double precision is required because pertubations of small amplitude often play a central role during plasma simulation. For the sake of simplicity, we focus here on the very classical linear Landau damping test case (with $k=0.5, \alpha=0.01$ ) which highlights the accuracy problem one can expect in Vlasov-Poisson simulation. The initial distribution function is given by $f\left(x, v_{x}, 0\right)=\frac{e^{-\frac{v_{x}}{2}}}{\sqrt{2 \pi}}(1+\alpha \cos (k x))$. Other test cases are available in our implementation, such as strong Landau damping, or two stream instability. They are picked to test the numerical algorithm and for benchmarking.

The problem arising with single precision computations is shown on Fig. 1. The reference LOss code (CPU version) is used here. The $L^{2}$ norm of electric potential is shown on the picture (electric energy) with logarithmic scale along the Y-axis. The double precision curve represents the reference simulation. The difference between the two curves indicates that single precision is insufficient; especially for long-time simulation. With an accurate look at the figure, one can notice that the double precision simulation is accurate until reaching a plateau value near $10^{-20}$. To go beyond this limit, a more accurate interpolation is needed. 


\subsection{Improvement of numerical precision}

For the time being, one has to consider mostly single precision (SP) computations to get maximum performance out of a GPU. The double precision (DP) is much slower than single precision (SP) on today's devices. In addition, the use of double precision may increase pressure on memory bandwidth.

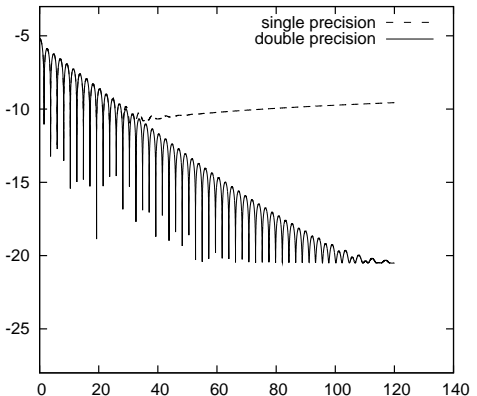

Fig. 1. Electric energy for Landau test case $1024^{2}$, single versus double precision (depending on time measured as a number of plasma period $\omega_{c}^{-1}$ )

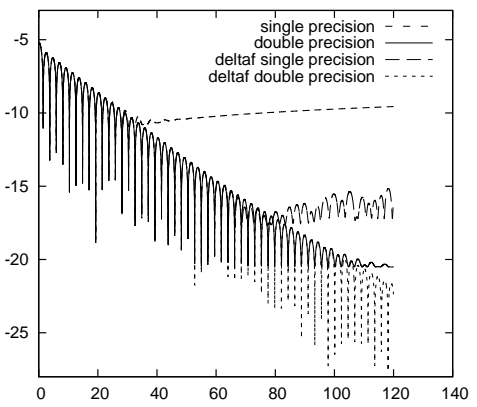

Fig. 2. Electric energy for Landau test case $1024^{2}$, using $\delta f$ representation or standard representation.

The previous paragraph shows that SP leads to unacceptable numerical results. It turns out that our numerical scheme could be modified to reduce numerical errors even with only SP operations during the advection steps. To do so, a new function $\delta f\left(x, v_{x}, t\right)=f\left(x, v_{x}, t\right)-f_{\text {ref }}\left(x, v_{x}\right)$ is introduced. Working on the $\delta f$ function could improve accuracy if the values that we are working on are sufficiently close to zero. Then, the reference function $f_{\text {ref }}$ should be chosen such that the $\delta f$ function remains relatively small (in $L_{\infty}$ norm). convenient to assume that $f_{\text {ref }}$ is a constant along the $x$ dimension. For the Landau test case, we choose $f_{\text {ref }}\left(v_{x}\right)=\frac{1}{\sqrt{2 \pi}} e^{-\frac{v_{x}^{2}}{2}}$. As the function $f_{\text {ref }}$ is constant along $x$, the $x$-advection applied on $f_{\text {ref }}$ leaves $f_{\text {ref }}$ unchanged. Then, it is equivalent to apply $\hat{x}$ operator either on function $\delta f$ or on function $f$. Working on $\delta f$ is very worthwile ( $\hat{x}$ operator): for the same number of floating point operations, we increase accuracy in working on small differences instead of large values. Concerning the $\hat{v_{x}}$ operator however, both $f_{\text {ref }}$ and $f$ are modified. For each advected grid point $\left(x, v_{x}\right)$ of the $f^{\star}$ function, we have ( $v_{x}^{o}$ is the foot of the characteristic):

$$
\begin{gathered}
f^{\star}\left(x, v_{x}\right)=f\left(x, v_{x}^{o}\right)=\delta f\left(x, v_{x}^{o}\right)+f_{\text {ref }}\left(v_{x}^{o}\right), \quad \delta f^{\star}\left(x, v_{x}\right)=f^{\star}\left(x, v_{x}\right)-f_{\text {ref }}\left(v_{x}\right), \\
\delta f^{\star}\left(x, v_{x}\right)=\delta f\left(x, v_{x}^{o}\right)-\left(f_{\text {ref }}\left(v_{x}\right)-f_{\text {ref }}\left(v_{x}^{o}\right)\right) .
\end{gathered}
$$

Working on $\delta f$ instead of $f$ changes the operator $\hat{v_{x}}$. We now have to interpolate both $\delta f\left(x, v_{x}^{o}\right)$ and $\left(f_{\text {ref }}\left(v_{x}\right)-f_{\text {ref }}\left(v_{x}^{o}\right)\right)$. In doing so, we increase the number of computations; because in the original scheme we had only one interpolation per grid point $\left(x, v_{x}\right)$, whereas we have two in the new scheme. In spite of this cost increase, we enhance the numerical accuracy using $\delta f$ representation (see Fig. 2). A sketch of the $\delta f$ scheme is shown in Algorithm 3. 


\section{CUDA ALGORITHMS}

\subsection{CUDA Framework}

Designed for NVIDIA GPUs (Graphics Processing Units), CUDA is a C-based general-purpose parallel computing programming model. Using CUDA, GPUs can be regarded as coprocessors to the central processing unit (CPU). They communicate with the CPU through fast PCI-Express ports. An overview of the CUDA language and architecture could be found in [NVI09]. Over the past few years, some success in porting scientific codes to GPU have been reported in the literature. Our reference implementation of Loss, used for comparisons, uses Fortran 90 and MPI library. Both sequential and parallel versions of LOss have been optimized over several years. The CUDA version of LOSS presented here mixes Fortran 90 code and external C calls (to launch CUDA kernels).
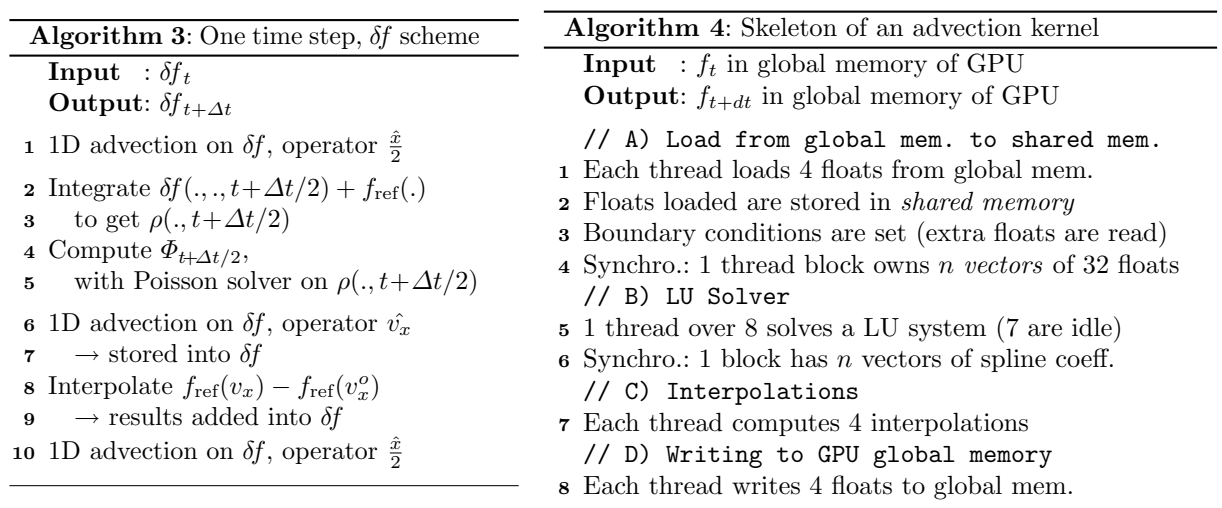

\subsection{Data placement}

We perform the computation on data $\delta f$ of size $\left(2^{j}\right)^{2}$. Typical domain size varies from $128 \times 128(64 \mathrm{~KB})$ up to $1024 \times 1024(4 \mathrm{MB})$. The whole domain fits easily in global memory of current GPUs. In order to reduce unnecessary overheads, we decided to avoid transfering $2 \mathrm{D}$ data $\delta f$ between the CPU and the GPU as far as we can. So we kept data function $\delta f$ onto GPU global memory. CUDA computation kernels update it in-place. For diagnostics purposes only, the $\delta f$ function is transfered to the RAM of the CPU at a given frequency.

\subsection{Spline coefficients computation}

Spline coefficients (of 1D discretized functions) are computed on patches of 32 values of $\delta f$. As explained elsewhere [CLS06], a smaller patch would introduce significant overhead because of the cost of first derivative computations on the patch borders. A bigger patch would increase the computational grain which is a bad thing for GPU computing that favors scheduling large number of threads.

The 2D domain is decomposed into small 1D vectors (named "patches") of $32 \delta f$ values. To derive the spline coefficients, tiny LU systems are solved. The 
assembly of right hand side vector used in this solving step can be summarized as follows: keep the 32 initial values, add 1 more value of $\delta f$ at the end of the patch, and then add two derivatives of $\delta f$ located at the borders of the patch. Once the right hand side vector is available (35 values), two precomputed matrices $L$ and $U$ are inverted to derive spline coefficients (using classical forward/backward substitution). We decided not to parallelize this small $L U$ solver: a single CUDA thread is in charge of computing spline coefficients on one patch That point could be improved in the future in order to use several threads instead of one.

\subsection{Parallel interpolations}

On one patch, 32 interpolations need to be done (except at domain boundaries). These interpolations are decoupled. To maximize parallelism, one can even try to dedicate one thread per interpolation. Nevertheless, as auxiliary computations could be factorized (for example the shift $v_{x} . d t$ at line 4 of Algo. 1), it is relevant to do several interpolations per thread to reduce global computation cost. The number of such interpolations per thread is a parameter that impacts performance. This blocking factor is denoted $K$.

\subsection{Data load}

The computational intensity of the advection step is not that high. During the $L U$ phase (spline coefficients computation), each input data is read and written twice and generates two multiplications and two additions in average. During the interpolation step, there are four reads and one write per input data and also four multiplications and four additions. The low computational intensity implies that we could expect shortening the execution time in reducing loads and writes from/to GPU global memory. So, there is a benefit to group the spline computation and the interpolations in a single kernel. Several benchmarks have confirmed that with two distinct kernels (one for building splines and one for interpolations) instead of one, the price of load/store in the GPU memory increases. Thus, we now describe the solution with only one kernel.

\subsection{Domain decomposition and fine grain algorithm}

We have designed three main kernels. Let us give short descriptions: KernVA operator $\hat{v}_{x}$ on $\delta f\left(x, v_{x}\right)$, KernVB adding $f_{\text {ref }}\left(v_{x}\right)-f_{\text {ref }}\left(v_{x}^{o}\right)$ to $\delta f\left(x, v_{x}\right)$, KernX operator $\hat{x}$ on $\delta f\left(x, v_{x}\right)$. The main steps of KernVA or KernX are given in Algorithm 4 . The computations of $8 n$ threads acting on $32 n$ real number values are described (it means $K=4$ hardcoded here).

First A) substep reads floats from GPU global memory and puts them into fast GPU shared memory. When entering the B) substep, all input data have been copied into shared memory. Concurrently in the block of threads, small $L U$ system are solved (but $87 \%$ of the threads stays idle). Spline coefficients are then stored in shared memory. In substep C), each thread computes $K=4$ interpolations using spline coefficients. This last task is the most computation intensive part of the simulator. Finally, results are written into global memory. 


\section{PERFORMANCE}

\subsection{Machines}

In order to develop the code and perform small benchmarks, a cheap personal computer has been used. The CPU is a dual-core E2200 Intel (2.2Ghz), $2 \mathrm{~GB}$ of RAM, $4 \mathrm{~GB} / \mathrm{s}$ peak bandwidth, 4 GFLOPS peak, $1 \mathrm{MB}$ L2 cache. The GPU is a GTX260 Nvidia card: $1.24 \mathrm{Ghz}$ clock speed, $0.9 \mathrm{~GB}$ global memory, $95 \mathrm{~GB} / \mathrm{s}$ peak bandwidth, 750 GFLOPS peak, 216 cores. Another computer (at CINES, FRANCE) has been used for benchmarking. The CPU is a bi quad-core E5472 Harpertown Intel ( $3 \mathrm{Ghz}), 1 \mathrm{~GB}$ RAM, $5 \mathrm{~GB} / \mathrm{s}$ peak bandwidth, 12 GFLOPS peak, L2 cache $2 \times 6 \mathrm{MB}$. The machine is connected to a Tesla S1070, 1.44Ghz, 4 GB global memory, $100 \mathrm{~GB} / \mathrm{s}$ peak bandwidth, 1000 GFLOPS peak, 240 cores.

\subsection{Small test case}

\begin{tabular}{|l|r||r|}
\hline Substeps in one time step & CPU (deltaf 4B) & GPU (deltaf 4B) \\
\hline \hline X Advection & $5123 \mu s(1.0)$ & $172 \mu s(29.7)$ \\
V Advection & $4850 \mu s(1.0)$ & $144 \mu s(33.7)$ \\
Field computation & $133 \mu s(1.0)$ & $93 \mu s(1.4)$ \\
\hline Complete Iteration & $10147 \mu s(1.0)$ & $546 \mu s(18.6)$ \\
\hline
\end{tabular}

Table 1. Computation times inside a time step and speedup (in parentheses) averaged over 5000 calls - $256^{2}$ Landau test case, E2200/GTX260

Let us first have a look on performance of the $\delta f$ scheme. We consider the small testbed (E2200-GTX260), and a reduced test case ( $256^{2}$ domain). The simulation ran on a single CPU core, then on the 216 cores of the GTX260. Timing results and speedups (reference is the CPU single core) are given in Table 1. The speedup is near 30 for the two significant computation steps, but is smaller for the field computation. The field computation part includes two substeps: first the integral computations over the 2D data distribution function, second a $1 \mathrm{D}$ poisson solver. The timings for the integrals are bounded up by the loading time of $2 \mathrm{D}$ data from global memory of the GPU (only one addition to do per loaded float). The second substep that solves Poisson equation is a small sequential 1D problem. Furthermore, we loose time in lauching kernels on the GPU ( $25 \mu s$ per kernel launch included in timings shown).

\begin{tabular}{|l|r||r||}
\hline $\begin{array}{l}\text { Substeps in one time } \\
\text { step }\end{array}$ & CPU (deltaf 4B) & GPU (deltaf 4B) \\
\hline X Advections & $79600 \mu s(1.0)$ & $890 \mu s(90)$ \\
V Advections & $89000 \mu s(1.0)$ & $1000 \mu s(89)$ \\
Field computation & $1900 \mu s(1.0)$ & $180 \mu s(11)$ \\
\hline Complete Iteration & $171700 \mu s(1.0)$ & $2250 \mu s(76)$ \\
\hline
\end{tabular}

Table 2. Computation time and speedups (in parentheses) averaged over 5000 calls - $1024^{2}$ Landau test case E2200/GTX260

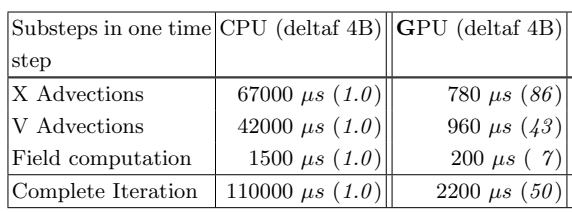

Table 3. Computation time and speedups (in parentheses) averaged over 5000 calls - $1024^{2}$ Landau test case Xeon/Tesla1070 


\subsection{Large test case}

In Tables 2-3, we look at a larger test case with data size equal to $1024^{2}$. Compared to a single CPU core, the advection kernels have speedups from 75 to 90 for a GPU card (using 260000 threads). Here, the field computation represents a small computation compared to the advections and the low speedup for the field solver is not a real penalty. A complete iteration reaches a speedup of 76 .

\section{CONCLUSION}

It turns out that $\delta f$ method is a valid approach to perform a Semi-Lagrangian Vlasov-Poisson simulation using only 32-bit floating-point precision instead of classical 64-bit precision. So, we have described the implementation on GPU of the advection operator used in Semi-Lagrangian simulation with $\delta f$ scheme and single precision. A very fine grain parallelization of the advection step is presented that scales well on thousands of threads. We have discussed the kernel structure and the trade-offs made to accommodate the GPU hardware.

The application is bounded up by memory bandwidth because computational intensity is small. It is well known that algorithms of high computational intensity are able to be efficiently implemented on GPU. We have demonstrated that an algorithm of low computational intensity can also benefit from GPU hardware. Our GPU solution reaches a significant speedup of overall 76 compared to a single core CPU execution. In the near future, we expect to have a solution for $4 \mathrm{D}$ semi-Lagrangian codes (2D space, $2 \mathrm{D}$ velocity) that runs on a GPU cluster.

\section{References}

$\left[\mathrm{BAB}^{+} 08\right]$ K. J. Bowers, B. J. Albright, B. Bergen, L. Yin, K. J. Barker, and D. J. Kerbyson. $0.374 \mathrm{pflop} / \mathrm{s}$ trillion-particle kinetic modeling of laser plasma interaction on roadrunner. In Proc. of Supercomputing. IEEE Press, 2008.

[CK76] C.Z. Cheng and Georg Knorr. The integration of the Vlasov equation in configuration space. J. Comput Phys., 22:330, 1976.

[CLS06] N. Crouseilles, G. Latu, and E. Sonnendrücker. Hermite spline interpolation on patches for a parallel solving of the Vlasov-Poisson equation. Technical Report 5926, INRIA, 2006. http://hal.inria.fr/inria-00078455/en/.

[CLS09] N. Crouseilles, G. Latu, and E. Sonnendrücker. A parallel Vlasov solver based on local cubic spline interpolation on patches. J. Comput. Phys., 228(5):1429-1446, 2009.

[LCGS07] G. Latu, N. Crouseilles, V. Grandgirard, and E. Sonnendrücker. Gyrokinetic semi-lagrangian parallel simulation using a hybrid OpenMP/MPI programming. In $P V M / M P I$, pages 356-364, 2007.

[NVI09] NVIDIA. CUDA Programming Guide, 2.3, 2009.

[SDG08] George Stantchev, William Dorland, and Nail Gumerov. Fast parallel particle-to-grid interpolation for plasma PIC simulations on the GPU. $J$. Parallel Distrib. Comput., 68(10):1339-1349, 2008.

[SRBG99] E. Sonnendrücker, J. Roche, P. Bertrand, and A. Ghizzo. The semilagrangian method for the numerical resolution of the Vlasov equations. J. Comput. Phys., 149:201-220, 1999. 